\title{
EDUCACIÓN INTERCULTURAL BILINGÜE: UNA EXPERIENCIA DIRECTA CON EL PENSAMIENTO AMAZÓNICO COCAMA-COCAMILLA EN LA COMUNIDAD NATIVA "INDEPENDENCIA" -RÍO AMAZONAS-LORETO.
}

\section{Bilingual Intercultural Education: A Direct Experience with The Cocama-Cocamilla Amazon Thought in The Native Community "Independencia"- Amazon River-Loreto.}

Ferdinand Luis Hagiwara Grández*

\begin{abstract}
RESUMEN
El presente artículo tiene como propósito reflexionar sobre algunos aspectos importantes de la Educación Intercultural Bilingüe, en base a una experiencia de investigación del pensamiento amazónico cocama-cocamilla en la comunidad nativa "Independencia" Río Amazonas-Loreto durante el año 2014, lo cual nos permitirá abordar algunos conceptos como el de saberes ancestrales, interculturalidad, pensamiento amazónico cocama-cocamilla, currículo oculto en la actividad educativa de la comunidad Independencia.
\end{abstract}

Palabras clave:

Educación Intercultural Bilingüe/ Pensamiento Amazónico cocama-cocamilla/ Cosmovisión Amazónica/ Currículo Oculto/ Filosofía de la Cultura/ Interculturalidad.

\begin{abstract}
The purpose of this paper is, to reflect on some important aspects of Bilingual Intercultural Education, based on a research experience of the cocama-cocamilla Amazonian thought, in the native community "Independencia" Río AmazonasLoreto during 2014, which will allow us to address some concepts such as ancestral knowledge, interculturality, Amazonian Thinking cocama-cocamilla, hidden curriculum in the educational activity of the "Independencia" community.
\end{abstract}

\section{Keywords:}

Intercultural Bilingual Education / Amazonian Thinking cocama-cocamilla / Hidden Curriculum / Interculturality.

* Docente de la Universidad Femenina Sagrado Corazón: ferdinandhagiwarag@unife.edu.pe 


\section{INTRODUCCION}

La Educación Intercultural Bilingüe relacionada con la recuperación de los "saberes ancestrales" en los pueblos originarios americanos, está sustentada en una Filosofía de la Cultura. Al respecto, David Sobrevilla nos dirá que "El uso figurado de la palabra "cultura", que tan importante nos ha llegado a ser, es en verdad relativamente tardío: aparece una vez en la antigüedad romana, en Cicerón, para luego desaparecer y volver a reaparecer [...] en la Época Moderna. Este surgimiento relativamente reciente del empleo figurado del vocablo cultura parece dar en principio razón a Heidegger, cuando sostiene que el concepto de cultura sólo pudo aparecer en el suelo nutricio del pensar moderno [...]" (Sobrevilla 2013:11).

El concepto de interculturalidad, entendida como respeto a las diferentes culturas, tolerancia a sus modos de vida y diálogo con sus formas de pensamiento, tendría que ser una expresión necesaria de la Educación Intercultural Bilingüe. Esta tendencia de respeto a las culturas se ha ido fomentando y desarrollando porque la historia nos ha enseñado el peligro del pensamiento único expresado en ideologías totalitarias, las que nos han llevado a hechos históricos lamentables (Primera y Segunda Guerra Mundial).

El pensamiento divergente como forma contraria al pensamiento único, que indica diversas maneras de mirar la realidad, sería en ese sentido una mejor manera de expresar la interculturalidad y su expresión a través de la Educación Intercultural Bilingüe, respetando, de esta manera, diferentes formas de ver la vida (cosmovisiones) y de comprender su significado (racionalidades).
Desde el punto de vista histórico, el cambio del pensamiento único al pensamiento divergente, implicó el cambio de paradigma de la modernidad al paradigma de la posmodernidad. La filosofía de la postmodernidad, según Gianni Vattimo, se expresaría como "pensamiento débil", entendiendo por él, la actitud de tolerancia y respeto hacia otras formas de ver el mundo, es decir, otras racionalidades y que sería contraria a la actitud etnocéntrica de imponer mi racionalidad a las demás, que se expresaba o expresaría como "pensamiento fuerte", muy propia de los procesos colonizadores realizados por las potencias europeas desde el siglo XV.

Esta nueva actitud nos permite comprender y aprender de pueblos que históricamente han sido excluidos y han estado silenciados en el proceso históricosocial de la colonización europea por ocupar un lugar de subordinación. Sin embargo, tales pueblos originarios tienen mucho que decirnos y enseñarnos a través de sus "saberes ancestrales". Y solo una actitud de apertura y respeto pudo revelarnos en la época actual estos saberes ancestrales tan vinculados con la "madre tierra" y tan respetuosa de la naturaleza, ya que el pensamiento único etnocentrista consideró a estos pueblos como carentes de cultura $y$, en consecuencia, nada se podría aprender de ellos.

\section{LOS PUEBLOS ORIGINARIOS Y LOS SABERES ANCESTRALES}

Los "saberes ancestrales" constituyen el bagaje cultural que permite a estos pueblos convivir en armonía con la naturaleza, esto es, respetando la tierra, cuidando del agua y preservando los bosques. En una palabra, con total respeto al entorno ecológico. Los pueblos amazónicos, pobladores de la mayor 
cuenca en biodiversidad del planeta y en reserva de agua, se constituyen en verdaderos maestros de la conservación del bosque, es decir, de la conservación de su entorno natural.

Son importantes, además, sus "saberes ancestrales" porque permiten aprovechar la flora y la fauna sin destruirla, pues ellos solo toman las plantas y animales que van a consumir en el día; no hay lugar para la acumulación o la explotación indiscriminada de las especies, pues para ellos, eso sería transgredir los principios que rigen la vida del hombre con la naturaleza.

Para comprender mejor lo que son los saberes ancestrales y cómo se vinculan con la vida de estos pueblos originarios Carvalho nos señala lo siguiente:

Se denominan [...] saberes ancestrales [...] a todos aquellos saberes que poseen los pueblos y comunidades indígenas, y que han sido transmitidos de generación en generación por siglos. Estos conocimientos, saberes y prácticas se han conservado a lo largo del tiempo principalmente por medio de la tradición oral [...], y también por medio de prácticas y costumbres que han sido transmitidos de padres a hijos en el marco de las dinámicas de la convivencia comunitaria que caracterizan a nuestros pueblos indígenas. [...]. Deberíamos primero comprender que los saberes ancestrales son la expresión de una cosmovisión, profunda y compleja, [...], en la que la intuición y el sentir se entrelazan con el pensamiento para generar el conocimiento del mundo. [...], podría decirse que los saberes ancestrales abarcan una gran variedad de aspectos del conocimiento y la técnica que van desde el lenguaje hasta la gastronomía, desde las matemáticas hasta la artesanía, pasando por la medicina, la construcción, la silvicultura, las técnicas de conservación del ambiente y microclimas, la producción y alimentación, la agricultura y el riego, el transporte y la comunicación, [...]. (Nicolás Carvalho En revista El Telégrafo del 13/09/2015)

\section{LA INTERCULTURALIDAD Y LA EDUCACIÓN INTERCULTURAL BILINGÜE}

Hoy en día, por lo menos once países (Argentina, Bolivia Brasil, Colombia, Ecuador, Guatemala, México, Nicaragua, Paraguay, Perú y Venezuela) reconocen y aceptan a través de sus Constituciones la interculturalidad, lo que deja traslucir el carácter "multinacional" de las comunidades nativas. Asimismo, otros países latinoamericanos se van sumando a este reconocimiento, haciéndose una tendencia mundial.

Para vincular las tendencias interculturales con una Educación Intercultural Bilingüe, debemos señalar que, hablar de Educación es hablar de la vida misma. La educación en su concepto más tradicional alude a la reproducción o retransmisión de la cultura de un pueblo desde las generaciones adultas hacia las más jóvenes. Deberíamos entonces preguntarnos ¿cuál es la educación que conviene a estos pueblos originarios?

El Ministerio de Educación del Perú, nos señala que "En el marco de una práctica pedagógica intercultural, las escuelas deben promover el fortalecimiento de los saberes de los pueblos teniendo en cuenta los procesos propios en los que 
se adquieren, considerando los tiempos y lugares en las que se desarrollan y los responsables de transmitirlos." (Rutas de Aprendizaje para la Educación Intercultural Bilingüe II Ciclo: 2014: 6)

Sin embargo, la educación oficial no ha estado a la altura de las necesidades de estos grupos étnicos, ni en concordancia con las características multilingües y pluriculturales de nuestro país. La explicación de ello es que la educación formal representa siempre los intereses de las clases dominantes o los grupos de poder heredados, en este caso, desde la colonización de América.

Al respecto Hagiwara (2014) nos señala que "las tendencias interculturales buscan recuperar los conocimientos de los pueblos ancestrales olvidados por mucho tiempo o minimizados hasta la nihilización por tendencias etnocentristas de origen europeo. [...], el encuentro entre europeos y americanos significó un choque cultural entre dos maneras de entender el mundo, dos racionalidades distintas que se ubican una encima de la otra por el solo hecho de implicar una relación de poder: vencedores y vencidos [...]."

El modelo capitalista neoliberal, que se transmite en la educación formal con los estilos de vida implicados, es considerado peligroso para las culturas de estos pueblos originarios, debido a las experiencias negativas que han tenido frente a la intromisión de transnacionales en sus respectivos territorios, pues han tenido repercusiones en lo social, motivando el desmembramiento familiar y la pérdida de su modelo de vida relacionado directamente con el entorno ecológico.

Encontramos que estos fueron intensamente explotados durante la expansión de la economía amazónica como "mano de obra barata" para la extracción del caucho y también durante el boom petrolero en la expansión de la economía amazónica. Pero aún más, al convertirse en trabajadores de las empresas petroleras, el modelo de vida tradicional fue desplazado, dando lugar a la incorporación del modelo consumista y a la pérdida de muchos valores culturales. Los hombres pasaron a integrar las empresas petroleras causando un impacto en su modelo tradicional, dando lugar a un modelo consumista con pérdida de muchos valores culturales. Asimismo, frente a la irrupción del oro negro y su explotación económica, sus ríos y lagos se vieron contaminados, no pudiendo reaccionar adecuadamente ante esta agresión de su entorno ecológico, por estar asimilados como trabajadores.

En este contexto, muchos de los Apus de estas comunidades consideran que es mejor "cerrarse" a la intromisión del mundo moderno por ser una amenaza real a la forma de vida que han tenido durante siglos, lo cual también desde nuestro punto de vista, no nos parece adecuado, porque afecta la verdadera interculturalidad. La tecnología, a través de los medios de comunicación, también pueden convertirse en algo peligroso, para la forma de vida que ellos tienen, porque los valores consumistas que propagan los medios televisivos, pueden constituirse en un lavado de cabeza para los jóvenes que querrán imitar esos estilos de vida que observan en la televisión.

\section{ASPECTOS HISTÓRICOS DE LA ETNIA}

Los primeros españoles que tuvieron contacto con estos grupos de la Amazonía, dentro de la conquista, 
están relacionados con el nombre de Juan Salinas de Loyola, en 1557, quien menciona la nación Cocama o la Gran Cocama, por su fama de haber tenido mucha gente y por el temor que inspiraba entre sus vecinos del Ucayali, cuando recorrían los ríos en armadas de cuarenta y cincuenta canoas. Salinas de Loyola, fue además el primer europeo en navegar el río Ucayali encontrando pueblos de entre 200 y 400 casas, que se extendían en el recorrido. El nombre de Gran Cocama quedaba así justificado. (El Ojo Verde: Cosmovisiones Amazónicas, 2015)

En otra etapa posterior, en la colonización, encontramos a los cocamas-cocamillas tratando de ser evangelizados por los jesuitas. Ellos realizaban su trabajo pastoral y evangelizador, reduciendo a la población indígena, en centros misionales, con la finalidad de catequizarlos e integrarlos a la religión cristiana. Paralelamente se buscaría aprovechar su fuerza laboral como se había realizado en la zona andina, pero esto no tuvo éxito, ya que el indígena amazónico, no estaba acostumbrado a vivir reducido en un modelo de aldea y estando a la par con distintos grupos étnicos, sino más bien, su modelo obedecía a un patrón de migración constante, donde por las características agrícolas de pobreza del suelo amazónico (lixiviación), tenían que ir rotando las tierras desplazándose en ciclos regulares. Entonces, el modelo jesuita, generaba una serie de epidemias por el hacinamiento, ocasionando muertes masivas, a lo cual respondieron con rebeliones, dando muerte a los misioneros, destruyendo las aldeas e internándose nuevamente en la floresta. (Ardito, 1993)

En el siglo XVIII y XIX, los procesos migratorios unidos a los auges económicos del barbasco (Lonchocarpus) y caucho (Castilloa ulei) generaron el boom demográfico y en consecuencia el desarrollo de las ciudades en la Amazonía. Llegaron muchos europeos quienes reclutaron mano de obra indígena, siendo una de las principales la mano de obra cocama. En ese proceso, muchos cocamas migraron a Brasil y otros cocamas andariegos fundaron comunidades por toda la Amazonía peruana. Hoy encontramos, por esta razón, cocamas en lugares tan distantes entre sí como Pucallpa (San Pablo de Tushmo), los alrededores de Iquitos (poblados de Santo Tomás, Rumo Cocha, Independencia y Santa Clara) y en el río Putumayo, frontera con Colombia. También están en Requena y Nauta, ciudades más antiguas y pobladas de Loreto. (Sunción, 2010)

\section{LA EXPERIENCIA DIRECTA: EN LA COMUNIDAD NATIVA "INDEPENDENCIA" LORETO RÍO AMAZONAS}

Visitamos "Independencia" cuatro veces. Utilizamos el método de triangulación concurrente que nos permitió confirmar o corroborar resultados y efectuar una validación cruzada entre datos cuantitativos y cualitativos (Ñaupas, 2014: 405).

Con respecto a la etnia, algunos datos de AIDESEP (Asociación Interétnica de Desarrollo de la Selva del Perú, 2009) nos señalan que este grupo pertenece al tronco Tupí-guaraní. Los datos estadísticos de los cocama-cocamilla como población total también nos señalan rangos variables entre 10,500 y 40,000 habitantes, en comunidades asentadas en Perú, Colombia y Brasil. En el Perú, están dispersos en diferentes comunidades en los cursos bajos de los ríos Marañón, Ucayali y Nanay, 
y también en el mismo Amazonas; como es el caso de "Independencia" otros puntos poblacionales están en caseríos cerca de Pucallpa, en el Bajo Huallaga y en el Urituyacu también en la Región Loreto. (INEI, 2007)

Datos acerca de la población de Independencia no existen en el INEI, pero calculamos un aproximado de 398 pobladores de todas las edades.

\section{PENSAMIENTO AMAZÓNICO COCAMA-COCAMILLA}

La Cosmovisión cocama-cocamilla recurre a la representación estratificada en diversos niveles. Según (Rivas, 2000) los mundos son considerados de la siguiente manera: Existe un primer sol (Wepe Kuarachi), en el mundo acuático donde viven seres como los kuarara (una especie de hombres del agua) con sus gorras que son rayas y sus casas de pura tierra. También allí vive la boa (muiwatsu) que bota burbujas al mundo de los peces, el tigre de agua que sujeta a la boa a petición de los kuarara, para que no salga de la cocha y no haya remolinos.

En un segundo nivel del mundo acuático con un segundo sol (Wepe Mukuika), viven los peces, lagartos, bufeos, el paiche y boas pequeñas. La Ipira Mama con sus largos cabellos es la dueña de éste mundo y ella decide que crezca o merme (disminuya) el agua.

En el tercer nivel con el tercer sol (Mutsapirka), vive el pueblo cocama con sus plantas, animales, seres naturales, curanderos y la gente que ha muerto. Quienes dominan este mundo son los espíritus del monte y a ellos se les pide permiso para sembrar, pescar o ir de cacería, solo así, los animales caerán en las trampas o habrá buena pesca.
En el cuarto nivel con el cuarto sol (Irakua), viven las almas y se relacionan con el médico ayahuasquero o chamán. En las cumbreras de las casas que se encuentran entre flores, estrellas y pájaros, viven las almas de los muertos buenos. Los muertos malos son quemados y sus cenizas forman las nubes del cielo.

En el quinto nivel con el quinto sol (Pichka), vive Dios y también está Kémari (El hijo de Dios). Más abajito, se encuentran los cerros y, elevado en medio de eso, vive el cóndor mama, el jefe de todas las aves de la tierra.

Fernando Santos Granero del SMITHSONIAN TROPICAL RESEARCH INSTITUTE señala que, en el caso de las cosmovisiones indígenas amazónicas, no sólo se plantea la existencia de una diversidad de mundos, cada uno con sus propias topografías, habitantes y leyes, sino la existencia de una diversidad de esferas al interior del mundo en que vivimos.

En efecto, un rasgo común a estas cosmovisiones es su concepción animista del universo, la cual postula que todo lo material, sea objeto o sujeto, tiene una contraparte espiritual. En el caso de los objetos, los fenómenos que llamamos "naturales", y los animales, su dimensión espiritual es concebida como una esencia primordial: la forma primera y verdadera que éstos tenían en el origen de los tiempos, antes de adquirir su apariencia actual. Estas esencias, que por lo general tienen forma humana, son parte integrante de las cosas, fenómenos y animales, pero suelen desprenderse y vagar por esta tierra. Además, existen en el mundo una serie de seres incorpóreos: divinidades, demonios, espíritus benévolos y malévolos que pueden adquirir una apariencia material 
y hacerse visibles, pero cuya esencia es espiritual e invisible.

Para los indígenas amazónicos la realidad material es una máscara, un disfraz, que oculta la "verdadera" realidad. No por ello, sin embargo, es una realidad "menos real". Tanto la apariencia material como la esencia espiritual son consideradas reales, pero mientras que la apariencia no es más que un "envoltorio" pasivo, a la esencia se le atribuyen poderes extra-ordinarios.

Los mundos invisibles, así como los seres espirituales y las esencias primordiales que los habitan, son depositarios de conocimientos y fuerzas místicas que son indispensables para el bienestar de los humanos y el buen funcionamiento de su sociedad. Por ello, un elemento central en la experiencia religiosa de los indígenas amazónicos, tanto en el ámbito personal como en el colectivo, es llegar a conocer la dimensión normalmente invisible de la realidad.

\section{EL CURRÍCULO OCULTO}

Torres (2005:10) señala que el curriculum oculto juega un papel destacado en la configuración de unos significados y valores de los que el colectivo docente y el mismo alumnado no acostumbran a ser plenamente conscientes. En este sentido, podemos darnos cuenta, que el currículo oculto, funciona a un nivel profundamente cultural y tiene que ver con las creencias de un colectivo que se socializan permanentemente, es decir, se reproducen socialmente al margen de currículos considerados oficiales, propios de la educación formal.

Al respecto, podemos mencionar que la Educación Intercultural Bilingüe no está ajena a este componente curricular y en el caso de los pueblos originarios, actúa poderosamente a través de los principios y valores que rigen el calendario comunal y que es parte de la vivencia y supervivencia de estos grupos. Fernández (2005) señala que el currículo oculto en el caso de los aymara del norte de Chile (Pachica y Arica) se da a través de cinco principios: Dualidad de pares opuestos-juego de parcialidades, Orden y armonía-"Taypi", Reciprocidad- Niveles de organización del trabajo. Redistribución (Ayni-mita-minkaQamasa), Pachakuti-ciclicidad (PachaQamasa-Ajaya-Sarayaña), JerarquíaAutoridad-Jiliri-Markachirinaka.

En el Tercer Principio: Reciprocidad - Niveles de organización del trabajo. Redistribución (Aynimita -minka Qamasa), el mismo Fernández describe: Mediante este principio se asume que el ser humano está dotado de diversas dimensiones, tanto en el sentido espiritual como material y que ello influye en su accionar en los otros ámbitos: sociales, religiosos, económicos, culturales, etc. Este valioso principio enseña el sentido del trabajo en equipo o en comunidad en donde cada uno es responsable de una parte de la misión, pero todo ello conduce mediante la organización natural al éxito que es, a la vez, un producto que se comparte y redistribuye. La aplicación Pedagógica de este principio, continúa Fernández, sería reconocer la importancia y el rol del alumno y la alumna tanto en su curso, como en su familia y en la comunidad en general, valorizar el descanso diario como una forma de distribuir correctamente las horas del día y disponerse para un nuevo esfuerzo diario, diferenciar y valorizar adecuadamente las actividades físicas o motrices de las mentales y espirituales reconociendo su importancia para el desarrollo armónico de la personalidad del niño. 
Resaltamos este tercer principio, mencionado por Fernández, porque en la Cosmovisión Amazónica se constituye en columna vertebral de la vida en comunidad; en ellos y en especial en los cocama-cocamilla se presentan unificados como principios de solidaridad, reciprocidad y redistribución, manteniéndose como eje de la vida comunitaria, donde lo material y lo espiritual se une y permeándose además a la vida educativa como currículo oculto, manifestándose en educadores, estudiantes y población en general.

Podemos señalar con Fernández (2005) que la educación formal y oficial, representante de la cultura dominante, no logró acallar las formas culturales propias, "no logró aniquilar formas educativas preexistentes en las culturas indígenas, aunque tuvieran que mantenerse en una forma de "currículum oculto", que con muchas dificultades lograrán mantener tales culturas. El desafío para los educadores en una sociedad intercultural consiste precisamente en rescatar esos valiosos saberes para transformarlos en aprendizajes previos que permitan iniciar un proceso educativo que, sin despreciar los valores de una educación externa, configure un currículum que integre y haga legítimos los elementos culturales y socioeducativos de los pueblos originarios de América, para ello se requiere de la voluntad y respeto recíproco de indígenas y no indígenas. Este desafío se enfrenta con dos ventajas fundamentales: por una parte, se cuenta con la participación activa de los mismos pueblos con todos sus elementos educativos espontáneos y, por otra, con los antecedentes de esfuerzos realizados en el pasado por algunos educadores."

\section{RESULTADOS}

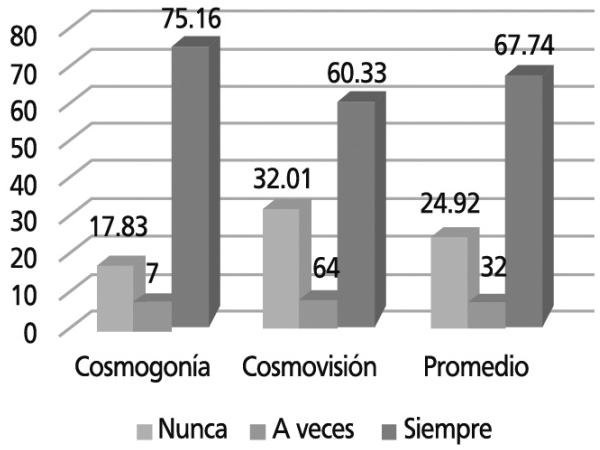

Figura 1

En la figura 1, podemos observar un índice del $75.16 \%$ con respecto a la Cosmogonía (creencias míticas sobre el origen del pueblo), que debemos tomarlo como altamente significativo. Con respecto a la Cosmovisión (Ideas y Opiniones sobre la vida) ocurre algo similar donde obtenemos un porcentaje promedio de $60.33 \%$. El promedio obtenido de ambos es de $67.74 \%$, lo que nos indica que pensamiento amazónico cocamacocamilla: Cosmogonía, Cosmovisión, es altamente significativo en su influencia sobre la Educación Intercultural Bilingüe en esta comunidad.

\section{CURRÍCULO FORMAL DE LA EDUCACIÓN INTERCULTURAL BILINGÜE}

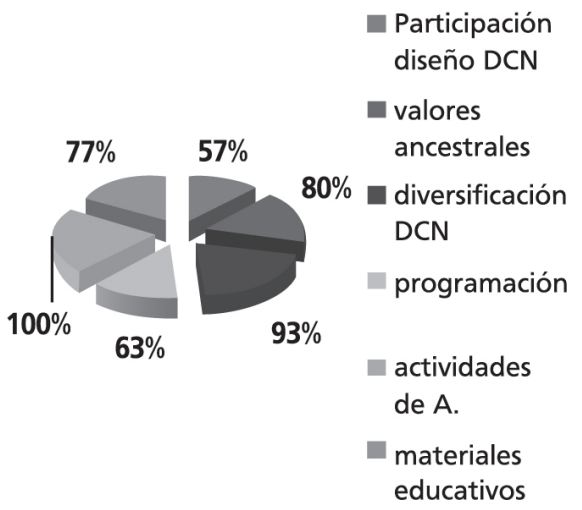

Figura 2 
En la figura 2, observamos que existe una participación del 57\% de la comunidad en internalización de contenidos del Diseño Curricular Nacional (DCN), participando luego en la contextualización a su realidad local en un $93 \%$, lo que se observa en la programación anual de los docentes en un 63\% y en el desarrollo de actividades de aprendizaje en un $100 \%$, utilizando materiales educativos en un $77 \%$. Por último, en un nivel altamente significativo de $80 \%$ se sienten identificados con sus valores ancestrales, lo cual es muy importante si tenemos en cuenta que han sufrido el menoscabo de estos valores a través de los medios de comunicación y de modelos contrarios a estos valores ancestrales.

\section{CURRÍCULO OCULTO COMUNIDAD NATIVA INDEPENDENCIA}

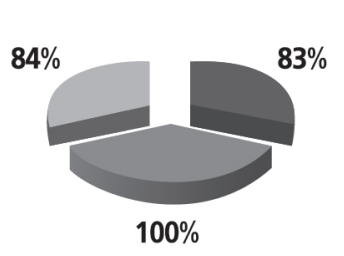

- Solidaridad

- Interdependencia

reciprocidad

Figura 3

En la figura 3, observamos que el currículo oculto expresado en los principios de Solidaridad, Interdependencia y Reciprocidad y que se manifiesta en la Educación Intercultural Bilingüe son altamente significativos, teniendo como resultados para el principio de reciprocidad $100 \%$, para el principio de solidaridad $84 \%$ y para el principio de interdependencia 83\%, lo que nos indica que la familia tiene un papel importante en la transmisión de los valores ancestrales que se expresan en la vivencia comunitaria.

\section{DISCUSIÓN DE RESULTADOS}

Los resultados a nivel descriptivo evidencian que existe un alto nivel de identificación entre los pobladores de la comunidad nativa "Independencia", con sus creencias costumbres y tradiciones, expresados en sus ideas y opiniones. Estos hallazgos, señalan que efectivamente, la cosmogonía y cosmovisión de los pueblos y nacionalidades indígenas amazónicas se encuentran en estrecha relación con el entorno/medio ambiente y con el mundo espiritual que forman parte de los "saberes ancestrales" que se concretizan en la realidad cotidiana de la comunidad.

En la cosmovisión de los cocamas, el conocimiento del universo amazónico es uno solo, no existe separación, pues, ambas explican la realidad. Ellos siguen creyendo que todo el universo es vivo y participan activamente en los eventos sociales bajo este pensamiento holístico. En cambio, el pensamiento llamado "occidental" ha parcelado la realidad entre "seres vivos" y "seres inertes"; supone que las montañas no tienen vida. De esta manera, separando la naturaleza, la llaman "recursos naturales" y no "madre tierra", influenciado por el pensamiento euro centrista, y que se reproduce a través de la escuela tradicional.

Efectivamente, podemos mencionar que los pobladores de la comunidad Independencia, realizan una selección de contenidos propuestos desde el Ministerio de Educación, en un sentido de contextualizarlos a su propia realidad y a partir de este "filtro", pueda llegar a sus niñas y niños, Sin embargo, se visualiza aún serias deficiencias en la educación formal y en el apoyo del Estado con textos adecuados a lo que sería una Educación Intercultural Bilingüe desde la necesidad real de la comunidad. Esta historia real, 
nos lleva a plantearnos, como sociedad inclusiva, la necesidad de construir una propuesta de Educación Intercultural y Bilingüe que profundice aún más en la importancia de los saberes y valores del pensamiento autóctono y surja de su propia realidad y de las reales necesidades y demandas de su población.

Por último, el elemento tradicional de sus valores está muy presente en ellos y se rescata a través del currículo oculto que en este caso acompaña y fortalece la práctica comunitaria, el respeto al bosque, la obtención de los alimentos a través de la pesca, la caza y la recolección de frutas que prodiga la naturaleza, pero que a su vez no debe romper el equilibrio con los espíritus del bosque, porque eso sería desastroso para la comunidad, donde el hombre es una pieza más en este mundo holístico e integrador donde el respeto a los espíritus del bosque y entidades tutelares es una obligación.

\section{CONCLUSIONES}

El acceso al conocimiento y vivencia del pensamiento amazónico cocamacocamilla nos permite comprender los elementos necesarios para desarrollar una Educación Intercultural Bilingüe acorde con sus necesidades de desarrollo social y calidad de vida.

A partir de esta experiencia, se debe valorar otras de pensamiento y racionalidad, teniendo en cuenta que el Perú es un país multiétnico y pluricultural y que, por lo mismo, la Educación Intercultural Bilingüe debe ser el elemento común y normal en las instituciones educativas de Costa, Sierra y Selva.

La consecuente discriminación, rechazo o minusvaloración de las etnias originarias, no estaría nada más que demostrando, el carácter nocivo del etnocentrismo cultural y la profunda ignorancia frente a lo que se constituye como verdadera riqueza de nuestro país: la pluriculturalidad.

Se debe garantizar a partir del Estado una Educación Intercultural Bilingüe de calidad por las características de nuestro país, reconocido en la Constitución Política del Estado. Solo de esta manera se vencerán barreras de exclusión y racismo y se incluirá efectivamente en el desarrollo nacional, saberes ancestrales y valores tradicionales de los cuales sentirnos orgullosos como peruanos.

Los contenidos del currículo oculto provienen de la diversidad de expresiones culturales propias, referidos a ideas, creencias, "saberes ancestrales" y formas de entender el mundo basados en principios, que se manifiestan en el desarrollo del programa educativo como observamos en el caso de la comunidad nativa cocama-cocamilla de "Independencia".

Las mejoras continuas en Educación Intercultural Bilingüe deberán nacer del diálogo permanente con los pobladores de estos pueblos originarios del Perú, en dirección a obtener un programa educativo con tejido propio, con matices diferenciados, con textura y color originales

\section{REFERENCIAS BIBLIOGRÁFICAS}

AIDESEP/ISPP (2009). Visiones Kukama-Kukamiria en relación al bosque y la sociedad. Programa de Formación de Maestros Bilingües de la Amazonía Peruana. Iquitos.

Ardito, W. (1993). Las reducciones Jesuitas de Maynas. Lima. Ediciones CAAAP 
El Ojo Verde: Cosmovisiones Amazónicas. Lima 2015

Hagiwara, F. (2014). Cosmogonía y cosmovisión en la racionalidad y el pensamiento cocama-cocamilla. Lima.

Revista de la UNIFE

MINEDU. Rutas del Aprendizaje para la Educación Intercultural Bilingüe II Ciclo. -Castellano Amazonía, Segunda Edición, junio 2014.

Ñaupas, H. Mejía, E. Novoa, E. Villagómez, A. (2014). Metodología de la Investigación:

Cuantitativa-Cualitativa y Redacción de la Tesis. Bogotá. Ediciones de la U.

Rivas, R. Ipurakari: los cocamacocamilla en la varzea de la Amazonía Peruana. Tesis doctoral presentada en la Pontificia
Universidad Católica del Perú, año 2000, Lima Perú.

Sobrevilla, D. (s/f). Introducción a la Filosofía de la cultura y al estudio de las culturas en el Perú.

Sunción, V.H. (2010). Antecedentes etnohistóricos de la fundación de Requena. Editorial del

Instituto de Investigaciones Educativas e Históricas de la Amazonía Peruana. Iquitos-Perú.

Torres, Jurjo. (2005). El Curriculum Oculto. Ediciones Morata. Octava edición. Madrid-España

http://dialogoandino.cl/wp-content/ uploads/2016/07/da-28-2006-03. pdf (recuperado el 05/06/2019) Mg. Emilio Fernández Canque: Una aproximación al conocimiento del "currículum oculto" en la educación indoamericana.

Recepción: 13 de junio del 2019 Aceptación: 09 de octubre del 2019 